\title{
CHAOTIC LIGHT: A THEORY OF ASYMMETRIC RESONANT CAVITIES
}

\author{
JENS U. NÖCKEL ${ }^{1}$ AND A. DOUGLAS STONE \\ Department of Applied Physics and Physics, Yale University \\ P. O. Box 8284, New Haven Connecticut 06520-8284 ${ }^{2}$
}

\begin{abstract}
Spherical and cylindrical dielectric cavities support high $\mathrm{Q}$ whispering gallery modes due to total internal reflection of the trapped light. When such a cavity is deformed smoothly the ray dynamics of these modes becomes chaotic in a manner determined by the KAM theory of classical hamiltonian dynamics. The universal properties of the ray dynamics predicted by KAM theory allow a general understanding of the whispering gallery modes of such asymmetric resonant cavities (ARCs). This theory combined with simulations of the non-linear map describing the ray motion provides the basis for a ray-optics model of the Q-spoiling of these whispering gallery modes for large deformations (greater than $1 \%$ of the radius). The model predicts a sharp onset as a function of deformation for significant Q-spoiling of these modes and highly directional emission above this threshold. Solution of the wave equation for typical whispering gallery modes confirms the qualitative behavior predicted by the ray-optics model even when the cavity is only a few times the resonant wavelength. The model explains for the first time the lasing intensity profile of highly deformed lasing droplets.
\end{abstract}

\footnotetext{
${ }^{1}$ Current address: Department of Physics, University of Oregon, 1371 E 13th Avenue, Eugene, OR 97403

${ }^{2}$ Published in Optical Processes in Microcavities, edited by R.K.Chang and A.J.Campillo (World Scientific Publishers, 1996)
} 


\section{Introduction}

\subsection{The Asymmetric Resonant Cavity (ARC)}

Optical processes in microcavities are currently under intensive study [1] with several goals: to design novel microlasers [2, 3, 4] and other optical devices [5], to study non-linear optical effects [6], and to investigate the interaction of atoms with single cavity modes [7]. The cavities employed in these investigations can be divided into two categories: Fabry-Perot type cavities of various designs, and dielectric cavities (spheres or cylinders) in which the surface of the dielectric confines certain modes of the electromagnetic field by total internal reflection. In the dielectric cavities these high-Q modes are the "whispering gallery" (WG) modes in which light rays circulate just inside dielectric surface, propagating almost tangent to it [8]. The research described below pertains exclusively to the whispering gallery modes of dielectric cavities. For obvious geometric reasons such high-Q modes are only possible when at least one cross-section of the cavity is bounded by a convex curve (i.e. a curve which has positive curvature everywhere) as is the case for a cylinder and a sphere. However we are not primarily concerned here with the WG modes of ideal dielectric spheres or cylinders but instead with the WG modes of a new class of resonant cavities which are created by deformations of these ideal symmetric dielectric cavities. The deformations considered can be quite large, ranging from 1-50\% of the undeformed radius; the crucial assumption is that they maintain the convexity of the cavities. While the WG modes of a spherical or cylindrical cavity can be treated analytically and the effect of small deformations included by perturbation theory [9], the deformations we consider are so large that the modes of the deformed cavities are not perturbatively related to those of the ideal cavity. Hence we shall refer to the class of deformed cavities we study as asymmetric resonant cavities (ARCs) since their properties are not derivable from those of the symmetric cavities (in any simple manner). Because the modes of ARCs are not simply related to those of symmetric cavities it is not immediately obvious that high-Q WG modes still exist in such structures; however we will show below that general principles of non-linear dynamics imply that under rather general conditions such modes exist if the cavity surface remains convex.

\subsection{ARCs and Quantum Chaos}

Because ARCs strongly violate at least one spatial symmetry of the undeformed cavity, the wave equation describing their modes or resonances is not separable in at least two of the three spatial dimensions and the solutions are not any of the familiar special functions of mathematical physics [10]. Instead the wave equation can only be solved numerically, e.g. by the T-matrix method [11] or by wavefunction matching techniques [12] of the type we use in subsection 7.2 below. It is for this reason that one does not find the properties of such cavities described in advanced optics textbooks or even in the research literature. Furthermore, brute force solution of the wave equation 
for a few modes in a given geometry usually does not yield much physical insight into the general properties of ARCs. However the properties of wave-equations with reduced or zero spatial symmetry have been extensively studied during the past decade in another context, the attempt to understand the quantum mechanics of systems which have either partially or fully chaotic classical dynamics. This field of inquiry is widely referred to by the unfortunate descriptor "quantum chaos", although it is now generally agreed that there exists no quantum analogue of the exponential sensitivity to initial conditions which is the hallmark of classical chaos. Considerable insight into such problems has been obtained using both semiclassical and statistical arguments. The semiclassical theory pioneered by Gutzwiller [13] differs substantially from the familiar Bohr-Sommerfeld quantization due to the chaotic nature of the classical orbits in such systems. The main applications of these ideas have been in atomic and solid-state physics (see for example Ref. 14) where detailed quantitative agreement between theory and experiment has been achieved in a number of cases. In each case the theoretical work has begun from an understanding of the chaotic classical mechanics. In perhaps the best-studied system, the hydrogen atom subjected to intense microwave radiation, many of the puzzling features of the variation of the ionization threshold with frequency and intensity can be understood on a wholly classical basis, by locating the transition point to (global) classical chaos [15]. The theory we develop below is similar to this work in spirit, although substantially different in detail [16]. In particular, in the present work we deal with a time-independent energy-conserving dynamical system whose transition to chaos is described by the Kolmogorov-Arnol'dMoser (KAM) theory of non-linear dynamics. Although it is not an exaggeration to term this theory one of the highest achievements of modern mathematical physics, it has had very few direct applications to experimental systems; we believe that this fact should lend interest to the experimental study of ARCs. The essential idea of the KAM theory and its application to ARCs will be explained in detail in section 3 below.

\subsection{Applications of ARCs}

Before beginning the description of the theory of ARCs let us mention the possible practical applications such resonators may have in optics or optoelectronics. Dielectric microspheres or microdisks already provide compact high-Q resonators which may be useful as spectral filters or as components in microcavity lasers. Based on the work to be presented below [17] we can propose three further advantages that ARCs may provide:

1. The ability to tune the Q-value and resonant frequency of the cavity by appropriate deformations.

2. If this can be done in situ, then the possibility of designing a Q-switched ARC micro-cavity laser. 
Figures available from author's website.

Figure 1: Ray trajectories for circle (a), and quadrupole-deformed circle (b) parametrized by $r(\phi)=1+\epsilon \cos 2 \phi$ in polar coordinates for $\epsilon=0.08$ corresponding to an $8 \%$ fractional deformation, or an eccentricity $e=0.56$. Rays are launched from the boundary at the same $\phi$ and angle of incidence $\sin \chi_{0}=0.7$ in both cases; ray escape by refraction only occurs in case (b).

3. The ability to couple a high-Q WG mode out of the cavity with strong directionality.

The third item is perhaps of most importance since the lack of intrinsic directionality in microdisk lasers has been recognized as a major barrier to their development for applications [18]. If ARCs can combine the advantages of microdisk or microsphere cavities with the directional emission found in Fabry-Perot resonators it will become possible to develop useful microlasers which can take full advantage of the reduction of the lasing threshold which arises as the number of cavity modes overlapping the gain region is decreased [1]. The current generation of semiconductor microlasers (the Vertical Cavity Surface-Emitting Laser) does not gain much of this advantage due to the fact that the lasing modes are only two-dimensionally confined. Of course obtaining a large threshold reduction requires a microcavity whose dimensions are comparable to the wavelength of light and the ray-optics model which will take up the bulk of this article does not apply when the wavelength approaches the cavity size. Nonetheless we have solid numerical evidence that the qualitative behavior predicted by the ray-optics model and in particular the directionality of emission are still present in this limit. For all these reasons we believe that ARCs represent a new class of cavity resonators whose properties and usefulness need to be explored in a series of controlled experiments on a well-characterized realization. As a first step we have recently applied the theory to explain the lasing intensity profile of deformed lasing droplets [19] (see section 8), but much more work is needed and appropriate solid ARCs need to be designed and studied.

\section{Physical Origin of Q-spoiling in ARCs}

\subsection{EM/QM Analogy}

As noted in the introduction, problems in quantum chaos are often studied with semiclassical methods. The analogue of the classical limit in quantum mechanics is the ray-optics limit $(\lambda \rightarrow 0)$ of the (Maxwell) wave equation and so we begin by considering the ray optics of a deformed cavity. For simplicity consider an infinite dielectric cylinder of radius $R$. In Fig. 1(a) we show the ray motion corresponding 
Figures available from author's website.

Figure 2: Effective potential picture for whispering gallery resonances; $k_{\min }=$ $m /(n R)$ and $k_{\max }=m / R$.

to a typical WG mode of a perfect cylinder in the (x-y) plane perpendicular to its axis. Note that as the ray circulates it always collides with the surface of the cylinder at the same angle of incidence; this is clear geometrically, but may also be regarded as a consequence of the conservation of the z-component of angular momentum in a rotationally symmetric potential. Therefore, if such a ray is trapped by total internal reflection at its initial bounce, it will remain trapped forever within the strict rayoptics approximation. Of course even if we ignore for the moment absorption or microscopic imperfections in the dielectric which may limit the lifetime, it is wellknown that such resonances do have a finite lifetime due to evanescent leakage. This may be seen clearly by invoking the similarity between the wave equation in quantum mechanics and electromagnetism [20]. If we assume an index of refraction $n=1$ outside the scatterer and $n>1$ but constant inside the dielectric, then in each of these regions the wave equation for the electric field has the form

$$
-\nabla^{2} \mathbf{E}+\left(k^{2}-n^{2} k^{2}\right) \mathbf{E}=k^{2} \mathbf{E}
$$

where $k=\omega c$ is the wavevector of the light in vacuum. Even though the matching conditions for $\mathbf{E}$ at the interface of the dielectric can be very different from the quantum mechanical ones for the wave function (depending on the polarization), one can still consider $V_{E M}(\mathbf{r})=\left(1-n^{2}(\mathbf{r})\right) k^{2}$ as the analogue of a potential. One sees therefore that the dielectric body acts like an attractive potential well for the scattering of EM radiation, and in general an attractive well does not give rise to very sharp resonances. However the occurence of sharp resonances for a cylindrical dielectric can be understood by noting that if one separates the wave equation in cylindrical coordinates $(\rho, \phi, z)$ then the equation for the radial variation of the electric field contains an effective potential $V_{\text {eff }}(\rho)=V_{E M}(\rho)+m^{2} / \rho^{2}$ where $m$ is an integer representing the conserved $L_{z}$ in the system. Hence the effective potential is the sum of the attractive well due to the dielectric and the repulsive $L_{z}$ angular momentum barrier, see Fig. 2. For wavevectors satisfying

$$
m /(n R)<k<m / R
$$

there exists a "classically allowed" region inside the dielectric separated by the angular momentum barrier from the outside region where propagation is also allowed. Thus sharp resonances are found in this interval of wavevector for this angular momentum channel; their width in the ideal system is determined by the "tunneling" (evanescent leakage) through the angular momentum barrier. 


\subsection{Classical Escape and Ray Chaos}

However this point of view makes it clear that these sharp resonances arise only due to conservation of $L_{z}$. In the same wavevector interval there exist broad resonances corresponding to lower $L_{z}$; a perturbation which destroys the rotational symmetry of the dielectric will mix these sharp resonances with this broad continuum and ultimately spoil their Q. The necessary mixing is not easily calculated perturbatively because the high-Q WG modes are separated in space from the lower Q modes and a weak perturbation will just mix different WG modes and not spoil their Q. Therefore it is useful to consider the question from the point of view of ray optics. In Fig. 1(b) we show the ray dynamics corresponding to a cylindrical dielectric with a $8 \%$ quadrupolar deformation in the plane perpendicular to its axis. The same initial angle of incidence $\sin \chi_{0}$ is used as in Fig. 1(a) but in this case, due to the deformation, the angle of incidence $\sin \chi$ is not conserved and fluctuates from bounce to bounce. This raises the simple question: will the angle of incidence fluctuate enough after a large number of bounces so that $\sin \chi$ will become smaller than $\sin \chi_{c}=1 / n$, allowing the ray to escape refractively according to Snell's law? If so, then intuitively one expects the Q of the corresponding WG resonances to be spoiled; if not there should still exist high-Q WG resonances at this deformation. Since a typical WG mode of the type shown in Fig. 1(a) will have a measured $\mathrm{Q}$ value $>10^{8}$ for a $10 \mu \mathrm{m}$ diameter cross-section this implies roughly $10^{6}$ collisions occur (at optical wavelengths) before escape. Therefore this "classical" Q-spoiling mechanism will become relevant if escape occurs in less than one million bounces; i.e. we are concerned here with the very long-time asymptotics of the ray motion. How can we determine when such classical Q-spoiling occurs?

The ray motion in the undeformed cylinder (Fig. 1(a)) is obviously confined by a circular caustic curve (a caustic curve is a curve which the ray touches at tangency once between each bounce). The presence of this caustic constrains the fluctuations in $\sin \chi$ (to be zero in this case). We will see shortly that for small deformations a dense set of caustics still exists and puts a bound on the fluctuations in $\sin \chi$ which is sufficient to prevent classical escape. The existence of caustic curves is characteristic of systems which have integrable (regular) dynamics or are weakly perturbed from integrability. The disappearance or "breaking" of these caustics is the hallmark of the hamiltonian transition to chaos for a free point mass bouncing specularly in a hardwalled cavity (in chaos theory such systems are referred to as "billiards" and when treated quantum-mechanically as "quantum billiards"). The ray dynamics problem of interest here is identical to such a billiard problem except for the possibility of refractive escape for sufficiently small values of $\sin \chi$ (we neglect evanescent leakage henceforth unless otherwise stated). For our problem the breaking of the caustics removes the bound on the fluctuations in $\sin \chi$ and allows the classical escape process and Q-spoiling of the WG modes. Hence in order to understand when and how this classical Q-spoiling occurs one must look to the theory of the hamiltonian transition to chaos, which is based on KAM theory [21]. The specific results on the breaking of 
caustics in convex billiards which are relevant here are due to Lazutkin [22].

\section{The Hamiltonian Transition to Chaos}

\subsection{Integrable and Chaotic Hamiltonians}

Before explaining the implications of KAM theory for our problem, we briefly review the concepts of integrability and chaos in hamiltonian systems [23]. A hamiltonian $H\left(\left\{q_{i}, p_{i}\right\}\right)$ is integrable if there exists a canonical change of variables to action-angle coordinates $\left\{a_{i}, J_{i}\right\}$ such that the new hamiltonian $H^{\prime}\left(\left\{a_{i}, J_{i}\right\}\right)=H^{\prime}\left(\left\{J_{i}\right\}\right)$, i.e. the energy only depends on the action variables $J_{i}$ and not on the angles $a_{i}$. For generic hamiltonians such a transformation does not exist; it is only for systems with a high degree of symmetry that such a change of variables can be found (unfortunately these systems represent almost all of the examples treated in textbooks, so they appear more common than they are). However if such a transformation exists then it immediately follows from hamilton's equations that all the $J_{i}$ are constants of motion $\left(\partial H / \partial a_{i}=0\right)$, and that the angles vary linearly with time (modulo $2 \pi$ ) with a frequency $\omega_{i}=\partial H / \partial J_{i}$. If we specialize to the two-dimensional case (which will apply to all the examples treated in this chapter), the phase-space of the motion is fourdimensional, with motion confined to three-dimensional surfaces of constant energy. However if the motion is integrable, then in fact any given phase-space trajectory lies on a two-dimensional subspace of the constant-energy surface specified by the conserved values of $J_{1}, J_{2}$. Since the angle variables are periodic, this subspace has the topology of a torus and the phase-space trajectory winds around this torus with $a_{1}=a_{1}(0)+\omega_{1} t, a_{2}=a_{2}(0)+\omega_{2} t$. If we define the winding number of a given torus as $W=\omega_{1} / \omega_{2}$ then the motion differs depending on whether $W$ is a rational or irrational number. If $W=p / q(p, q$ integer $)$ then each trajectory on that torus will close on itself after $p q$ windings and we have an infinite family of periodic orbits on that torus, no one of which covers densely the entire torus. On the other hand, if $W \neq p / q$ the orbit will never close on itself and each orbit will cover the entire torus quasi-periodically. Thus for the integrable system phase-space is divided up into nested rational and irrational tori corresponding to these two cases. We will see that for the relevant integrable billiards each irrational torus defines a caustic curve.

Independent of whether the motion is on a rational or irrational torus, if the system is integrable this motion is not ergodic, i.e. a typical phase-space trajectory does not visit all regions of the constant energy surface with equal density as $t \rightarrow \infty$, because each trajectory lives on a 2D subspace of this 3D surface. Now suppose an additional term is added to the hamiltonian which breaks its symmetry and destroys its integrability. It turns out that for sufficiently large perturbations in many cases there are no approximate conservation laws and typical trajectories starting anywhere in phase-space explore the entire 3D constant energy surface ergodically. Moreover any two trajectories corresponding to nearby initial conditions separate from one 
Figures available from author's website.

Figure 3: Integrable shapes (left) in two and three dimensions and their nonintegrable deformed counterparts.

another exponentially in time (up to some time scale). This situation is referred to as hard chaos. However KAM proved that for sufficiently weak non-integrable perturbations there still exists some remnant of the conservation laws present in the integrable limit and in particular that there exist many families of trajectories confined to a 2D subspace of phase-space (which thus are obviously not ergodic). It is best to explain this point in detail in the context of several examples relevant to ARCs.

\subsection{KAM billiards}

For the application to ARCs we wish to consider two-dimensional or three-dimensional families of billiards which are generated by deformations of circles or spheres, see Fig. 3) (the 2D case will describe cylindrical dielectrics with deformed cross-section, the z-motion being decoupled). In each case the size of the deformation will be specified by a continuous parameter which gives a circle or sphere when set equal to zero. We call these $K A M$ billiards because increasing the shape parameter from zero generates a KAM/Lazutkin transition in the dynamics. It is simplest to specify these billiards by giving the radius as a function of the relevant angle ( $\phi$ in the $2 \mathrm{D}$ case, $\theta$ in the 3D case (where we will focus on the systems with azimuthal symmetry). We can think of each such deformation as being a sum of multipole distortions, the simplest being the dipole and quadrupole distortions, which in 2D take the form:

$$
\begin{aligned}
& r(\phi)=\frac{1}{\sqrt{1+\epsilon^{2} / 2}}(1+\epsilon \cos \phi) \quad \text { (dipole) } \\
& r(\phi)=\frac{1}{\sqrt{1+\epsilon^{2} / 2}}(1+\epsilon \cos 2 \phi) \quad \text { (quadrupole) }
\end{aligned}
$$

The deformation parameter here is the constant $\epsilon$ multiplying the dipole or quadrupole term (the prefactors insure that the area remains constant and equal to $\pi$ ). The dipole billiard was first introduced by Robnik in order to study the quantum KAM transition [24]. Note that the dipolar distortion, to leading order in $\epsilon$, corresponds to a translation of the center of mass of the billiard which does not change its internal ray dynamics; when expanded around its new center of mass the distortion is primarily quadrupolar but without the four-fold symmetry of the quadrupole billiard. Since the dipole (Robnik) billiard becomes non-convex at smaller fractional deformations it shows a higher degree of chaos (for the same deformation) than the quadrupole 
Figures available from author's website.

Figure 4: Definition of the coordinates for the Poincaré section of 2D billiards.

billiard. In contrast, it is known [25] that an exactly elliptical deformation of the circular billiard does not generate chaotic dynamics at all: there still exists a constant of motion, not the angular momentum with respect to the center, but rather the product of the angular momenta with respect to the two foci, so the motion is still integrable. We may use the ellipse as a "control" shape to test the importance of chaos in the behavior of deformed cavities. Finally, axially symmetric deformations of the sphere can also be expressed in terms of a 3D multipole expansion of Legendre polynomials $P_{l}(\cos \theta)$ (an example is illustrated in Fig. 3); this type of description will be used to model deformed liquid droplets in section 8 below.

To compare the different 2D shapes, we introduce the eccentricity in analogy to the ellipse,

$$
e \equiv \sqrt{r_{\max }^{2}-r_{\min }^{2}} / r_{\max }
$$

where $r_{\max }$ and $r_{\min }$ are the extremal radii measured from the center of mass. The figures in the following sections will use this parametrization whereas we will find it helpful in the discussion to specify the fractional deformations in percent.

\subsection{The Poincaré Surface of Section}

Given the shape of the billiard and the specular reflection assumption, following the trajectory of specific rays is a simple exercise in geometry. However it is not always straightforward to detect structure in the phase-space motion simply by looking at the real-space trajectory. On the other hand the actual phase space trajectory typically occurs on a high-dimensional manifold which is difficult to visualize. Thus it is standard in non-linear dynamics to observe the phase-space motion by imaging the particle/ray every time it passes through a specified plane in phase-space; the resulting plot is called a Poincaré surface of section (SOS). For billiards it is simplest to choose the SOS to correspond to the boundary, with coordinates corresponding to the angular position $\phi$ around the boundary (since the billiards are convex there is only one boundary point for each angle), and the tangential component of momentum at the bounce point which is proportional to $\sin \chi$, the angle of incidence (see Fig. 4). There exists a non-linear (twist) [23] map $T_{\epsilon}$ which maps the vector $\left(\phi_{n}, \sin \chi_{n}\right)$ at the $n^{\text {th }}$ bounce to $\left(\phi_{n+1}, \sin \chi_{n+1}\right)$ at the next bounce. For the case of $2 \mathrm{D}$ billiards this map is typically defined implicitly by the roots of a third, fourth or fifth order polynomial. For every trajectory the coordinates $(\phi, \sin \chi)$ are plotted at each bounce (up to some number of bounces, e.g. 1000) giving a series of points in a 2D SOS plot (see Fig. 5); whether or not these points form a connected curve depends on the nature of the phase-space motion. It is usual to plot many trajectories in a single SOS in order 
Figures available from author's website.

Figure 5: Poincaré surfaces of section for the circle (a) as well as quadrupolar deformations with eccentricities $e=0.51$ (b) and $e=0.63$ (c). Vertical and horizontal two-bounce orbits in (a), shown as crosses and stars, are depicted in the schematic below the SOS. Shown to the right of each SOS are trajectories starting at $\sin \chi_{0}=0.7$ in all cases. In the schematics below (b), trajectories close to the horizontal and vertical diametric orbits are plotted (each below its bounce position in the SOS).

to image fully any phase-space structure which will prevent a single trajectory from visiting all regions. Initially we will neglect the possibility of ray escape in discussing the SOS.

The series of SOS's shown in Fig. 5 for the case of the quadrupole billiard as the deformation is increased clearly illustrates the content and implications of the KAM/Lazutkin theory. For zero deformation the SOS has two types of structures, solid horizontal lines and sets of discrete points arrayed on a horizontal line (we have denoted these sets of points by large symbols in the figure in order to make them more visible, but each corresponds to a single pair of bounce coordinates $(\phi, \sin \chi))$. Each solid line is the image of one quasiperiodic trajectory with irrational winding number, since these trajectories never close after many bounces they have visited the entire boundary (all values of $\phi$ ) and they yield a full line in the SOS. Note from the realspace picture of these orbits that such an orbit does generate a (circular) caustic. The trajectories which generate discrete sets of points in the SOS are the periodic orbits with rational winding number. The simplest of these are the two-bounce diametral orbits, two of which are denoted by plus and asterisk signs in Fig. 5(a). There are an infinite family of such orbits created by rotation of any one; each of these orbits are marginally stable, which means that nearby (non-periodic) orbits neither oscillate around the periodic orbit nor diverge from it exponentially. One such periodic orbit does not trace out a caustic curve but simply generates an inscribed polygon of the circle (e.g. a square for the 4-bounce orbit denoted by the squares).

Fig. 5(b) shows the SOS for the quadrupole billiard with $\epsilon=.065$ corresponding to a $6.5 \%$ fractional deformation; in this case the vertical diameter has been shortened and the horizontal one stretched. Now there are three types of structures in the SOS: closed curves which do not span the SOS (islands), grainy (chaotic) regions which are two-dimensional and have no apparent structure, and curves which span the SOS roughly horizontally (although they are no longer straight horizontal lines). The islands arise from periodic orbits which have been stabilized by the deformation. Stability in this context means that nearby orbits oscillate around the central periodic orbits. For example, since the vertical diameter has been shortened the vertical two-bounce orbit is now stable (this corresponds to the well-known fact in resonator theory that two confocal spherical mirrors separated by less than twice their radii of 
curvature are stable). Thus we see islands corresponding to nearby oscillatory orbits around the two-bounce vertical periodic orbit [the pluses in Fig. 5(a)].

In contrast, the stretching of the horizontal diameter has destabilized the twobounce horizontal periodic orbit; nearby orbits diverge from it exponentially (see inset) and give rise to a $2 \mathrm{D}$ chaotic layer in the SOS. The fact that this layer is twodimensional is important; no longer are single trajectories confined to points or lines on the SOS. This is the beginning of chaos, with exponential divergence of nearby trajectories and single trajectories exploring a finite fraction of the entire phase-space. Note that both the islands and the chaotic layers appear near periodic orbits of the unperturbed system; trajectories in that neighborhood are not perturbatively related to those of the undeformed billiard (a problem which was discovered by Poincaré in 1899 [26]). However, as noted above, there still exist full 1D curves spanning the SOS in the deformed case. These correspond to the (deformed) quasi-periodic orbits, which still trace out only a one-dimensional curve in the SOS which is only deformed from the unperturbed straight line. Thus these orbits are not ergodic and do not explore a finite fraction of the constant-energy surface. KAM theory proves that for sufficiently weak perturbations such quasi-periodic orbits still exist. These unbroken "KAM curves" partition phase-space so that a chaotic trajectory above a given curve in the SOS can never jump below that curve. For example in Fig. 5(b) one sees an unbroken KAM curve with a minimum of $\sin \chi=0.5$ and a maximum value of $\sin \chi \approx 0.65$; no ray starting with $\sin \chi_{0}>0.65$ can reach values of $\sin \chi<0.5$ so for an index of refraction $n=2\left(\sin \chi_{c}=1 / n=0.5\right)$ no classical escape is possible for a large set of WG orbits. Thus the unbroken KAM curves constrain the "diffusive" motion in the chaotic regions to lie in layers bounded by two KAM curves. Associated with each such "KAM curve" in the SOS is a caustic in the real-space motion which prevents rays from penetrating into the interior of the cavity, as seen in the inset. Lazutkin [22] proved that for any 2D convex cavity with a sufficiently smooth boundary such caustics exist, although as the cavity is very deformed from the circle these caustics only persist very near the boundary and may not constrain the relevant rays.

Fig. 5(c) shows the SOS for slightly higher deformation, $\epsilon=0.1$. The increased deformation has increased the degree of chaos in the motion and now unbroken KAM curves exist only very near the boundary $(\sin \chi>0.9)$; the bulk of the SOS is spanned by a chaotic region. A ray starting with $0.7<\sin \chi_{0}<0.9$ will typically be in this chaotic region and will diffuse to any value of $\sin \chi<0.9$ over some time scale. If as above $\sin \chi_{c}=0.5$ then such a ray will escape according to Snell's law (an example is shown in the inset). A SOS for $\epsilon>1 / 5$ (for which the quadrupole is non-convex) would show no KAM curves or islands, only a uniform sea of chaos covering the entire SOS.

\section{Ray Optics Model for Q-spoiling of ARCs}




\subsection{Classical Q factor}

The qualitative behavior of the ray dynamics of ARCs depicted in Fig. 5 is universal for all such smooth deformations of the circle (this is guaranteed by Lazutkin's theorem). That is why it is possible to find general physical principles that apply to all ARCs. What implications does this picture have for the whispering gallery modes of ARCs?

First, intuitively one associates WG modes with rays starting at $\sin \chi_{0}$ close to unity (i.e. rays moving almost tangent to the boundary). Whereas conventional geometric optics treatments for nonresonant scattering [27] start out with an incident beam, our method deals exclusively with rays escaping from inside the cavity. This will allow predictions about intrinsic properties of the resonant state that do not depend on the input coupling, such as resonance lifetime and directional lasing emission. In the eikonal approximation for the modes of a dielectric cylinder (see subsection 7.1) it is possible to associate an ensemble of rays having a fixed value of $\sin \chi$ with a mode of angular momentum index $m$ and wavevector $n k$ (within the dielectric) by the relation:

$$
\sin \chi=\frac{m}{n k R} .
$$

For the deformed cylinder no such simple relation exists (this is a key issue in the semiclassical quantization of chaotic systems [13]), but as a rough starting point for a ray-optics model of ARCs we will use Eq. (6) to connect the ray dynamics to the modal properties of ARCs. We have checked that our main conclusions are insensitive to this assumption. With this assumption we can define a ray-optics procedure for estimating the Q-factor of ARCs.

- Assign an index $m$ to the ARC mode which evolves by continuous deformation from the mode with angular momentum $m$ and wavevector $k$ in the undeformed cylinder.

- Associate this ARC mode with an ensemble of rays which is uniformly distributed in $\phi$ but has starting angle of incidence chosen by the eikonal rule: $\sin \chi_{0}=$ $m / n k R$.

- Propagate this ray ensemble forward in time and compute the mean classical escape rate $\tau^{-1}$, where the classical escape probability is zero for $\sin \chi>1 / n$ and unity for $\sin \chi<1 / n$.

- Define the quality factor associated with this ray ensemble as $Q=c k \tau$.

\subsection{Qualitative Predictions}

Our conjecture is that for dielectric ARCs with $n k R \gg 1$ the "classical" approximation for the Q-factor of mode $m$ is a good means for estimating the actual width of 
Figures available from author's website.

Figure 6: Escape rate for the dipole and the quadrupole with refractive index $n=2$ and starting condition $\sin \chi_{0}=0.8$. The escape rate is proportional to $1 / Q$. The plot assumes $n k R=100$; to convert to other size parameters, multiply the vertical scale by $100 /(n k R)$.

the resonance of index $m$ for deformations that are too large to be treated perturbatively. The universal phase-space evolution described above allows one to make certain qualitative predictions about the behavior of this classical Q-factor with increasing deformation:

1. For modes corresponding to a given $\sin \chi_{0}>\sin \chi_{c}$, Q will be infinite (the escape rate will be zero) up to some threshold deformation $\epsilon_{c}$ at which the last KAM curve intervening between $\sin \chi_{0}$ and $\sin \chi_{c}$ breaks. (In fact there will be some large but finite $\mathrm{Q}$ due to tunneling which we neglect).

2. For deformations $\epsilon>\epsilon_{c}$ the $\mathrm{Q}$ of these modes will be finite and decreasing as $\left(\epsilon-\epsilon_{c}\right)^{-\alpha}$ where the exponent $\alpha$ is in the range $2.5<\alpha<3.5$.

3. Once classical escape becomes possible the emission from these modes is highly directional and occurs (in the near field) only at the points where the regions of chaos in the SOS connect $\sin \chi_{0}$ to $\sin \chi_{c}$.

The first prediction follows clearly from the discussion in subsection 3.3; the second and third will be justified in more detail below.

\section{Threshold Behavior and Power Law}

Numerical evaluation of the classical approximation for $\mathrm{Q}$ for any simple model of 2D ARCs is straightforward; it just requires simulations of the same type used to generate the SOS's in Fig. 5, except here we only start ray trajectories at the value of $\sin \chi_{0}$ of interest and escape is allowed at a chosen $\sin \chi_{c}$. The mean inverse length $L^{-1}$ of the escaping trajectories is then computed and used to evaluate $Q=n k L$. Representative results for the dipole and quadrupole ARCs are shown in Fig. 6. As predicted, $Q^{-1}=0$ up to a threshold deformation (3\% for the dipole, $7 \%$ for the quadrupole) and then rises steeply. Note the threshold is not universal and is lower for the dipole ARC because of its stronger chaos compared to the quadrupole ARC for the same eccentricity.

In Fig. 7 we plot the data in Fig. 6 on a log scale vs. the log of the distance from threshold. As predicted these give a nice power law with an exponent $\alpha$ which is non-universal but in the range $2.5-3.5$. Briefly, the origin of this power law variation 
Figures available from author's website.

Figure 7: Power-law behavior of the escape rates in the previous figure, above the respective threshold deformations. Asterisks and dashed line are the data and linear fit for the dipole, squares and solid line refer to the quadrupole. The law $1 / Q \propto$ $\left(e-e_{c}\right)^{\alpha}$ is seen to hold, where $\alpha_{d}=2.6$ for the dipole and $\alpha_{q}=3$ for the quadrupole.

of $\mathrm{Q}$ from threshold is the following. In the relevant cases just below threshold there is one last KAM curve which blocks the phase-space flux from reaching the critical angle. As this last intervening KAM curve breaks with increasing deformation it leaves behind a "sticky" region in phase-space known as a cantorus[23] (because it has the properties of a Cantor set). The slow traversal of this cantorus is the rate limiting step in the classical escape process. Therefore the dependence of $\mathrm{Q}$ on the distance from threshold is determined by the mean phase-space flux through such a cantorus. This problem has been studied in detail and it is well-established that this flux varies as $\left(\epsilon-\epsilon_{c}\right)^{\alpha}$ with $\alpha$ in the range noted [28]. Space limitations do not allow us to reproduce those arguments here. However we note that the appearance of a powerlaw here is not surprising as the breaking of KAM tori constitutes a kind of critical phenomenon in phase-space, with the scale-invariance associated with second-order transitions [29].

\section{Ray Directionality}

As noted above, we expect the ray escape from ARCs to be directional leading to directional emission from deformed WG modes. Figure 8 shows the directional emission from the quadrupole ARC, obtained from a ray bundle started along $\sin \chi_{0}=0.83$ and escaping at $\sin \chi_{c}$. The simulation was performed for two different indices of refraction corresponding to $\sin \chi_{c}=0.5$ and $\sin \chi_{c}=0.65$. The histograms in the figure are obtained by sorting the escaping rays into bins according to the angle $\phi$ at which they escape; this should represent the near-field intensity distribution. The inset depicts the expected far-field intensity pattern taking into account the refraction of the escaping rays. The near-field histogram in both cases shows no escape whatsoever outside two very narrow regions around $\phi=0$ and $\phi=\pi$. The very high degree of directionality found in these simulations is initially somewhat surprising and deserves further study. To our knowledge, escape directionality has not been studied in the theory of phase space transport in Hamiltonian systems described briefly in section 3 because the systems previously considered were closed; although somewhat related issues have been studied in the context of chaotic classical scattering [30].

At the most general level the occurence of preferential escape directions and their location can be understood by simple curvature arguments. Rays total internally reflected from a point of high curvature are unlikely to escape at the next bounce since 
Figures available from author's website.

Figure 8: Directionality for the quadrupole at deformation $e=0.58$. The histograms shows the probability distribution of escape positions $\phi$, assuming $\sin \chi_{c}=0.5$ (solid) and $\sin \chi_{c}=0.65$ (hollow). The inset gives the resulting far-field directionality for $\sin \chi_{c}=0.5$, taking into account refraction on exit from the dielectric. The starting condition is $\sin \chi_{0}=0.83$.

Figures available from author's website.

Figure 9: Partial surface of section, only for $\sin \chi$ above the critical line for escape $\left(\sin \chi_{c}=0.5\right.$, shown dashed). The shape is the same as in Fig. 8. The heavy line with arrows is the tangent invariant curve of a corresponding ellipse superimposed on the quadrupole SOS (see subsection 6.2).

this will likely occur at a point of lower curvature and $\sin \chi$ will have increased. Since the converse is not true, rays are more likely to escape in the regions of high curvature. In the quadrupole these regions are near $\phi=0, \pi$ corresponding to the emission peaks in Fig. 8. However we shall see that such arguments alone cannot account for the narrowness of the peaks, their detailed structure, and their persistence in the far-field (as shown in the inset to Fig. 8). For example, for $\sin \chi_{c}=0.65$ there is no ray escape precisely at $\phi=0, \pi$, the points of maximum curvature.

First let us address the far-field behavior. Even if all rays escape very near $\phi=0, \pi$ the emission pattern in the far-field need not be highly directional since they might escape with very different angles of incidence. This does not occur since the phase space diffusion in the $\sin \chi$ direction is slow; rays approach the critical angle in small steps and escape very near $\sin \chi_{c}$, leading to almost tangential emission (see Fig. 5(c)). Therefore the far-field ray pattern is just as directional as the near-field escape histogram, but is simply rotated by $\pi / 2$ (again, see inset to Fig. 8).

\subsection{Ergodic model}

As a first step to understanding the escape directionality distribution we formulate a simple model which should correctly represent the short-time ray dynamics near the critical angle. In Fig. 9 we show the SOS corresponding to the escape histogram in Fig. 8 with the islands of stability shown, but the chaotic region left empty. One sees that there is a relatively large chaotic region developed around $\sin \chi_{c}$. Since motion in the chaotic region is ergodic (on that region) this suggests that as a starting point we can assume that the rays in the ensemble starting near $\sin \chi_{0}$ will after some time fill the chaotic region above $\sin \chi_{c}$ approximately uniformly. To test this notion we see if the observed directionality can be reproduced by starting with a homogeneous distribution of starting conditions above $\sin \chi_{c}$ and iterating the map one step (allow 
Figures available from author's website.

Figure 10: Escape directionality for $\sin \chi_{0}=0.85$ and $e=0.58$ The broad solid histogram is predicted by the simple ergodic model, the dotted line results from the modified ergodic model (see text). The heavy line is obtained from the invariant curve model. The full ray simulation leads to the most sharply peaked distribution (solid line).

each ray to collide once more with the boundary). The probability density $P(\phi)$ for escape in an interval $\mathrm{d} \phi$ will then just be proportional to the area of the SOS mapped in one step from above to below $\sin \chi_{c}$ and into the interval $\mathrm{d} \phi$. We call this set of assumptions the ergodic model for directionality. In order to recover the full symmetry of the billiard shape, we have to repeat this with an ensemble below $-\sin \chi_{c}$ (i.e. reversed starting momenta) and add the outcomes. The resulting approximation for the distribution $P(\phi)$ is shown in Fig. 10.

Although the ergodic approximation overlaps well with the peaks in the true escape probability density, the $P(\phi)$ it predicts is clearly much broader than the exact ray-tracing distribution. Since the ergodic model includes the short-time effects of the varying curvature around the boundary, this indicates that the directionality of escape from ARCs is not simply obtained from a knowledge of curvature. Obviously the assumption of a uniform distribution of rays filling phase-space above $\sin \chi_{c}$ has missed some essential part of the physics. What has been missed is that even in the chaotic component there is a definite flow pattern in phase-space for short times. This flow pattern is close to the KAM curves of the ellipse (which is integrable and has KAM curves at all deformations). Examples of such KAM curves are given in Figs. 9 and 11 where representative trajectories of the quadrupole billiard are found to follow them closely. Since for short times motion is along those curves most of the escape occurs near where these curves intersect the critical line at $\sin \chi_{c}$. Due to this flow pattern, many initial conditions (above $\sin \chi_{c}$ ) which lead to escape in the ergodic model can only be reached if the previous reflection occurred with $\sin \chi<\sin \chi_{c}$, implying that the ray would already have escaped before getting to the assumed starting point in phase space. One can begin to take this into account within an extended ergodic model by assuming zero occupation probability for initial conditions above $\sin \chi_{c}$ which upon one-step iteration backwards are below $\sin \chi_{c}$ (and uniform probability for all other initial conditions). Fig. 10 shows that this extension of the ergodic model significantly improves the predicted $P(\phi)$ as compared to the true distribution.

The advantage of the ergodic model is that only one or (in the extended version) two mapping steps need be considered. For the simple version it is then possible to express $P(\phi)$ in terms of the one-step map. The exact one-step map is not usually known in simple analytic form for KAM billiards, but we have been able 
Figures available from author's website.

Figure 11: Flow of three trajectories for the quadrupole at $e=0.57$, followed for 200 bounces each. The solid lines are the superimposed invariant curves of an ellipse with the same eccentricity.

Figures available from author's website.

Figure 12: Illustration of the areas $A$ and $\bar{A}$ which end up below or come from above $\sin \chi_{c}$, respectively, in one mapping step.

to develop reasonable approximate analytic expressions in certain cases [31]. If we abbreviate $p \equiv \sin \chi$, the map can be described by the two functions $\bar{\phi}(\phi, p), \bar{p}(\phi, p)$, giving the new position and momentum as a function of the old variables. The map is area-preserving, i.e. the Jacobian of the transformation $(\phi, p) \rightarrow(\bar{\phi}, \bar{p})$ is unity. One can alternatively specify the map by considering the old momentum and the new position as given so that the dependent variables are $\phi(\bar{\phi}, p)$ and $\bar{p}(\bar{\phi}, p)$. The differential probability of obtaining $\phi^{\prime}$ after one mapping step applied to a homogenous starting distribution with $p>p_{c}$ is

$$
P_{1}\left(\phi^{\prime}\right)=\frac{1}{|A|} \int_{A} d \phi d p \delta\left(\phi^{\prime}-\bar{\phi}(\phi, p)\right),
$$

where $A$ is only the region above $p_{c}$ that gets mapped below $p_{c},|A|$ is its area, and $\bar{\phi}$ is the new position after one reflection. The image of $A$ under the map, $\bar{A}$, has the same area $|\bar{A}|=|A|$ due to area preservation. These regions are shown in Fig. 12 . We make a transformation of variables in the integral from $\phi, p$ to $\bar{\phi}, \bar{p}$, yielding

$$
\begin{aligned}
P_{1}\left(\phi^{\prime}\right) & =\frac{1}{|\bar{A}|} \int_{\bar{A}} d \bar{\phi} d \bar{p} \delta\left(\phi^{\prime}-\bar{\phi}\right) \\
& \equiv \frac{1}{|\bar{A}|} \int_{\min \left[\bar{p}\left(\phi^{\prime}\right)\right]}^{\max \left[\bar{p}\left(\phi^{\prime}\right)\right]} d \bar{p} \\
& =\frac{1}{|\bar{A}|}\left(\max \left[\bar{p}\left(\phi^{\prime}\right)\right]-\min \left[\bar{p}\left(\phi^{\prime}\right)\right]\right) .
\end{aligned}
$$

Here, $\min \left[\bar{p}\left(\phi^{\prime}\right)\right]$ denotes the smallest $\bar{p}$ within $\bar{A}$ at the final angle $\bar{\phi}=\phi^{\prime}$, analogously $\max \left[\bar{p}\left(\phi^{\prime}\right)\right]$. The boundary of $\bar{A}$ is formed by the two curves $\left(\bar{\phi}, p_{c}\right)$ and $\left(\bar{\phi}, \bar{p}\left(\bar{\phi}, p_{c}\right)\right)$. In our case, $\bar{p}\left(\bar{\phi}, p_{c}\right)$ is a unique function of $\bar{\phi}$, and as a result $\max \left[\bar{p}\left(\phi^{\prime}\right)\right]=p_{c}$, $\min \left[\bar{p}\left(\phi^{\prime}\right)\right]=\bar{p}\left(\phi^{\prime}, p_{c}\right)$. We therefore have the simple result that

$$
P_{1}\left(\phi^{\prime}\right)=\left\{\begin{array}{lr}
\frac{1}{|A|}\left(p_{c}-\bar{p}\left(\phi^{\prime}, p_{c}\right)\right) & \text { for } p_{c}>\bar{p}\left(\phi^{\prime}, p_{c}\right) \\
0 & \text { otherwise. }
\end{array}\right.
$$


The distribution in Fig. 10 is obtained by forming the symmetrized function $P\left(\phi^{\prime}\right)=$ $\left[P_{1}\left(\phi^{\prime}\right)+P_{1}\left(-\phi^{\prime}\right)\right] / 2$.

For the extended ergodic model, we need the function $p(\phi, \bar{p})$. Then the lobes of $\left(\phi, p\left(\phi, p_{c}\right)\right)$ above $p_{c}$ delimit the region $A$. To decide whether a given $(\phi, p) \in A$ would have come from the region $p<p_{c}$ in the previous reflection, we simply invert the momentum $p$ and ask whether the forward mapping yields a new momentum $\bar{p}$ smaller in magnitude than $p_{c}$. This is true for a region $B$ bounded above $p_{c}$ by the curve $\left(\phi,-p\left(\phi,-p_{c}\right)\right)$. The modified starting domain for the ergodic model is then $A^{\prime}=A \backslash B$.

Whereas reasonable agreement with the near-field directionality of the full simulation can be obtained in the extended ergodic model, we do not expect correct results for the far-field which is seen in Fig. 8 to display the same degree of directionality. Since the ergodic models yield a final distribution of escaping trajectories that scatters widely in $\sin \chi$ (see $\bar{A}$ in Fig. 12), any near-field directionality will be spread out after refraction is taken into account. We thus need to understand why in fact only $\sin \chi \approx \sin \chi_{c}$ occurs upon escape.

\subsection{Tangent Invariant Curve Model}

The ergodic models discussed above have limited validity because they do not take fully into account the detailed structure of phase-space motion. That is the existence of a dominant flow pattern over many bounces and the persistence of stable islands and unstable periodic orbits (which repel phase-space flux). If there is a stable island which is intersected by the critical line $\sin \chi_{c}$ then no classical escape can occur in the interval of $\phi$ within the island. For example, for the particular shape giving the SOS of Fig. 9 there exists two large islands centered at $\sin \chi \approx 0.65, \phi=0, \pi$. These islands completely block escape at the points of highest curvature $\phi=0, \pi$, giving the surprising peak-splitting shown for this case in Fig. 8. The ergodic model on the other hand would find escape at those points from starting rays which could never have gotten into the island in the first place. The blocking of ray escape by remaining stable islands is a major effect in the lasing droplets which we study in section 8. There are also interesting effects in the directionality associated with unstable fixed points, but we cannot discuss them in detail here. These effects arise from stable and unstable periodic orbits and are less universal since different ARCs will have substantially different important orbits; however they can be understood on an individual basis if one is interested in a particular ARC.

Nonetheless there is still one further universal feature which relates to the directionality distribution of all ARCs which have a large quadrupolar component to their deformation. As noted above, the ellipse, which is integrable, is for small deformations primarily a quadrupolar deformation of the circle. Therefore all ARCs with a large quadrupolar component generate short-time ray dynamics close to the invariant curves of the ellipse as shown in Fig. 11. The existence of chaos in these 
ARCs allows motion perpendicular to these curves which would be forbidden in the ellipse, but for reasonable deformations this motion is rather slow. Thus, even when the SOS looks rather structureless and chaotic in the vicinity of $\sin \chi_{c}$ one finds as shown in Fig. 11 that the ray dynamics over hundreds of bounces follows the nearest invariant curve (IC) of the ellipse. Thus a reasonable representation of the motion is slow diffusion between these ICs until the IC which is tangent to the critical line is reached and then rapid escape near the points of tangency of this IC (which we call the tangent IC). This explains why most escaping rays do so at $\sin \chi \approx \sin \chi_{c}$. This description will only be good if $\sin \chi_{0}$ is separated from $\sin \chi_{c}$ by more than the width of the IC in $\sin \chi$, but this is typically the case for the WG modes of interest to us here. This picture of the phase-space motion can be tested by comparing $P(\phi)$ for our standard starting conditions to the $P(\phi)$ obtained by starting a uniform distribution of points on the tangent IC. In the latter case of course almost all the rays escape after a few tens of reflections, whereas many thousands are required in our ray-optics model. One finds that the tangent IC starting conditions reproduce the ray-optics $P(\phi)$ with remarkable accuracy (see Fig. 10). This finding has strong implications. First it implies that the directionality distribution of emission from the WG modes of ARCs is a short-time property, insensitive to the many thousands of reflections needed to reach the vicinity of the tangent IC. Therefore the directionality distribution is approximately independent of the initial conditions over a wide range of WG trajectories. This strongly suggests that all deformed $W G$ modes will tend towards the universal directionality distribution determined by the tangent $I C$ at a given index of refraction. If this is the case then the directions of highest emission from ARCs will be independent of the particular resonant mode excited, a property which should be quite important for applications, e.g. in a multi-mode laser.

\section{Wave Properties of ARCs}

\subsection{Eikonal Theory for Undeformed Case}

The ray optics model which we have developed in the previous sections relies on an eikonal (semiclassical) prescription introduced in subsection 4.1 to relate the mode indices of a WG resonance to the initial $\sin \chi$ of a ray ensemble. Unfortunately, because the semiclasical quantization of a mixed phase-space is still an open problem [32], we only have such a rule for the undeformed case; we now explain the origin of this rule. Since normally the WG resonances are very narrow even in the deformed case a good starting point should be the spectrum of the isolated cavity (i.e. with perfectly reflecting walls). The actual resonances in the leaky dielectric are frequency-shifted with respect to the isolated modes but the resulting corrections to $\sin \chi_{0}$ become negligible for large $k R$. The WG modes have been quantized semiclassically [33] for circular or elliptical cylinders, and for spheres. The method employed in that work can be also be applied to deformed cavities as long as the ray dynamics still exhibits 
Figures available from author's website.

Figure 13: (a) Integration path leading to Eq. (14), shown as the jagged solid line around the caustic $\mathrm{C}$ (dotted). Wavefronts of $\mathrm{S}_{C B}$ (dotted arcs) are perpendicular to all the rays (dashed straight lines) between $\mathrm{C}$ and the boundary $\mathrm{B}$. The continuation of the rays through $\mathrm{C}$ would not be normal to the wavefronts shown, but instead to their mirror images. (b) Exact resonance positions (dots) for the circular cylinder. The heavy line belongs to $m=k R$. The inset to (b) shows the scattered intensity from a plane wave into the angle element at $50^{\circ}$ from the beam axis, in the size parameter interval delimited in the main figure by horizontal dashed lines. The index of refraction is $n=2$.

a dense set of caustics, as shown in Fig. 13(a), however we do not attempt such an extension here. The eikonal approximation attaches to each ray a set of wavefronts; to describe the phase of these wavefronts we need to find the eikonal S(r) such that $\nabla \mathrm{S}$ gives the ray direction at all points $\mathbf{r}$. Assume a dense set of caustics of the ray motion exist, that is closed curves which the ray touches at tangency once between each reflection from the boundary. There are two rays going through every $\mathbf{r}$ in the region between caustic (C) and boundary (B), namely one coming from $\mathrm{C}$ to $\mathrm{B}$ and one reflected from B toward C. The eikonal expression for the electric field is a sum of two wavefronts,

$$
E(\mathbf{r})=A_{B C}(\mathbf{r}) e^{i n k \mathrm{~S}_{B C}(\mathbf{r})}+A_{C B}(\mathbf{r}) e^{i n k \mathrm{~S}_{C B}(\mathbf{r})} .
$$

When following a ray between two reflections from $\mathrm{B}$, we move first perpendicular to the surfaces of $\mathrm{S}_{B C}=c(c=$ const $)$, until we reach the caustic. Then we get from $\mathrm{C}$ back to $\mathrm{B}$ with the wave fronts $\mathrm{S}_{C B}=c$. In Fig. 13(a) only one half of this ray path is depicted for a number of starting points. In both halves of the ray path, the value of the phase $c$ increases by the length $l$ traveled along the ray between $\mathrm{B}$ and $\mathrm{C}$, because for any two points $\mathbf{r}_{1}$ and $\mathbf{r}_{2}$

$$
c\left(\mathbf{r}_{2}\right)=c\left(\mathbf{r}_{1}\right)+\int_{\mathbf{r}_{1}}^{\mathbf{r}_{2}} \nabla \mathbf{S}(\mathbf{r}) d \mathbf{r} .
$$

Using the latter relation, one obtains the desired quantization condition by following an alternative integration path that always runs perpendicular to the wave fronts of, say, $\mathbf{S}_{C B}$ and in addition forms a closed loop: Starting at $\mathbf{r}_{1}$ we can move on the wavefront to the caustic without accumulating any phase in (13). We then follow a ray for an infinitesimally small length to a neighboring wavefront of $\boldsymbol{S}_{C B}$ and move back to the caustic along this new wavefront, see Fig. 13(a). Repeating this procedure, one eventually encircles the caustic without ever crossing it, accruing a total phase equal to $L_{C}$, the length of the caustic, before reaching the end point $\mathbf{r}_{2}=\mathbf{r}_{1}$. In order 
for the function $E(\mathbf{r})$ in Eq. (12) to be single valued at $\mathbf{r}_{1}$, this phase difference must give unity when put in the exponential, i.e. we require

$$
n k L_{C}=2 \pi m \quad(m=0, \pm 1, \pm 2, \ldots) .
$$

For a circular domain of radius $R$, the length of the caustic is related to the classical angle of incidence $\chi$ by $L_{C}=2 \pi R \sin \chi$. Combining these results, we arrive at

$$
\sin \chi=\frac{m}{n k R}
$$

which is the semiclassical expression we have used above. It can be seen that the integer $m$ is precisely the angular momentum quantum number of quantum mechanics if we recall that the angular momentum of the photon at each bounce from the boundary is $M=\mathbf{r} \times(\hbar n \mathbf{k})=R \hbar n k \sin \chi=m \hbar$. Note also that a large number of resonances - those with approximately the same ratio $m / k R=n \sin \chi$ - are described by the same classical ray dynamics. Equation (15) can now be inserted into the condition for total internal reflection, $1>\sin \chi>1 / n$, to obtain

$$
n>\frac{m}{k R}>1 \text {. }
$$

This is identical to the condition given in Eq. 2, and agrees well with numerical solutions of the wave equation for the undeformed case. This is illustrated in Fig. 13(b) and (c); the only sharp resonances observed in the open cavity are those satisfying $k R<m$. Figure $13(\mathrm{c})$ shows the resonances in a window of $k R=10 \ldots 12$. Comparing with Fig. 13(b) where the same window is indicated by the two horizontal dashed lines, we see that the three narrowest resonances correspond to the three points with largest $m /(k R)$; and in general, the larger $m / k R$, the narrower the resonance.

The standard eikonal approximation does not allow us to calculate the width in the open cavity which is a tunneling effect. However it is possible to develop a WKB-type approximation for the small tunneling width in the limit of large $k R$ but at constant $m / k R[34]$ :

$$
\delta k=\frac{1}{2} \frac{1}{n^{2}-1} \exp [-(2 m-1)(\alpha-\tanh \alpha)]
$$

where $\tanh \alpha \equiv \sqrt{1-(k R)^{2} / m^{2}}$ is a constant between zero and one if we consider only rays of a fixed $\sin \chi$ (similar expressions are known for the Mie resonances of spherical dielectrics [20]). As we go to higher $k R$ and $m$, the width is seen to decrease exponentially. In order to understand the $\mathrm{Q}$ of ARC resonances we now ask how this narrow width changes as the cylinder is deformed.

\subsection{Exact solutions for the deformed cylinder}


Figures available from author's website.

Figure 14: Exact results for resonance positions and widths in the dipole as a function of deformation $e$. Left column: azimuthal mode index $m=20$, radial node number $n_{r}=0$; right column: $m=34, n_{r}=1$ (indices refer to $e=0$ ). Top : resonance positions; bottom: resonance widths (logarithmic scale). The solid lines are the widths as calculated from ray-optics simulation.

\subsubsection{Resonance widths}

In the inset to Fig. 13 it was assumed that a plane wave is incident normal to the cylinder. To solve this scattering problem, one uses polar coordinates $r, \phi$ and performs a partial wave analysis, decomposing the incoming plane wave into

$$
e^{i k x}=\sum_{m=-\infty}^{\infty}(-i)^{m} e^{i m \phi} J_{m}(k r) .
$$

Then one can solve the resulting decoupled radial equations for each $m$. These equations are obtained by imposing the matching conditions for the fields at the dielectric interface, noting that the field inside must be a superposition of Bessel functions $J_{m}(n k r)$, whereas the outgoing scattered wave consists of Hankel functions $H_{m}^{(1)}(k r)$. One can now try to satisfy the same matching conditions for the inside and outgoing waves alone, leaving out the incident wave. This is possible only at discrete complex values of the wavenumber, $k-i \kappa\left[\kappa>0\right.$ if we assume a time dependence $e^{-i \omega t}$ in the wave equation], and the corresponding solutions are the quasibound states of the cavity. For symmetric cavities and perturbative deformations their role in microcavity optics has been studied in detail previously (see for example Ref. 9. Resonances of width $\kappa$ occur at wavenumber $k$ when a quasibound state exists close to the real axis at $k-i \kappa$.

When the rotational symmetry is broken, the matching equations for different $m$ become coupled so that we have to solve a progressively larger system of simultaneous equations for the coefficients of the $J_{m}(n k r)$ inside and $H_{m}^{(1)}(k r)$ outside of the cavity. However the resulting quasibound state solutions evolve continuously out of those for the unperturbed cylinder as the deformation increases. This simplifies the search for resonances in the complex plane because we can start at the known quasibound state positions of the circular cylinder and then follow each solution as it moves through the complex plane when we change the shape. Representative results for the real positions and widths of WG resonances ar shown in Fig. 14. The size parameters $k R$ we have studied at this point are not large enough to expect the resonances to be accurately described by the ray-optics model; nonetheless the figure shows clearly a threshold behavior in the resonance width as expected from the classical model. The width remains small and almost constant up to a critical deformation, beyond which 
Figures available from author's website.

Figure 15: Comparison of exact wave results for dipole (crosses), quadrupole (asterisks) and ellipse (circles). The resonance considered is $m=20, n_{r}=0$ (in the undeformed case). The arrows indicate the classical thresholds for dipole (D) and quadrupole (Q).

a rapid broadening is found. One also sees that above the classical threshold the rayoptics model appears to give order-of-magnitude agreement with the width (note that there are no free parameters in this comparison). However for the resonances treated in Fig. 14 this broadening begins before the classical Q-spoiling threshold deformation is reached and has an approximately exponential dependence on the distance from threshold (and not the power-law predicted by the ray-optics model). We attribute this behavior to tunneling effects which will always be important near threshold because rays approach closely the critical angle. This would explain both the reduction of the threshold deformation and the exponential-like behavior (note that Eq. (17) predicts exponential variation of the width with $\sin \chi$ in the undeformed case).

Clearly at these values of $k R$ tunneling and interference corrections to the rayoptics model must be included. Such corrections then lead us into the true subject matter of quantum or wave chaos, and much more work needs to be done to reach a full semiclassical understanding of mixed phase-space quantum systems. Nonetheless we expect that as $k R$ increases these non-classical effects will become weaker and the ray-optics predictions will be more closely obeyed. For example, in the droplets studied in section $8, k R$ is at least an order of magnitude larger than in Fig. 14. The results of Fig. 14 do however raise the question of whether at the moderate size parameters $k R \sim 10-25$ investigated the classical mechanics of the cavity is relevant at all to the $Q$ of its WG resonances. In order to answer this question we compare in Fig. 15 the resonance widths of the three different types of cylindrical deformations introduced so far: elliptical (which is integrable), quadrupolar which exhibits a KAM transition to chaos, and the dipolar (Robnik) deformation which at equal fractional deformation exhibits more chaos than the quadrupole, as discussed in section 3. In all three cases a sort of threshold behavior is seen with initial exponential broadening above threshold. However the slope of this broadening differs substantially in the three cases with the most chaotic dipole ARC showing the most rapid broadening, the less chaotic quadrupole ARC having an intermediate slope, and the non-chaotic elliptical ARC showing the slowest broadening. At an eccentricity of 0.5 the dipole and elliptical WG modes corresponding to the same undeformed resonance differ in width by almost two orders of magnitude! This suggests that the rapid breakup of caustics which occurs in the dipole ARC and does not occur at all in the elliptical $\mathrm{ARC}$ has an important influence on the $\mathrm{Q}$ value of their resonances even at these moderate wavevectors. 


\subsubsection{Wave directionality}

To test the predictions of section 6 for the directionality of emission from a deformed cavity, we have to choose the proper boundary conditions in the wave equation corresponding to the situation of a uniform ray ensemble starting inside the dielectric and then escaping. This emission process differs from a scattering experiment which requires an incoming wave to excite the resonance. The directionality pattern in a scattering experiment will depend on the form of the incident wave both because of interference with the outgoing wave, and because the incident wave may couple preferentially to different senses of circulation of the rays. These effects are absent in emission, so a unique directionality profile will be observed that depends only on the quasibound state itself and should be approximately described by our ray optics model if the size parameter is sufficiently large.

If the resonant state is at the complex frequency $\omega-i \gamma \equiv c(k-i \kappa)$, then the corresponding solution of the time dependent wave equation decays at a rate $\gamma$ since it has the form $E(\mathbf{r}, t)=E(\mathbf{r}) e^{-i \omega t} e^{-\gamma t}$ where $\gamma>0$. But as a function of $r$, the outgoing waves in fact exhibit exponential growth because

$$
H_{m}^{(1)}(x) \approx \sqrt{\frac{2}{\pi x}} e^{i(x-m \pi / 2-\pi / 4)}
$$

for large values of $x=(k-i \kappa) r$. The physical reason for this growth with $e^{\kappa r}$ is a retardation effect: the field at $r \gg R$ has propagated away from the cavity where it originated a time $\Delta t \approx r / c$ in the past - but at that earlier time the field at the cavity was larger by a factor $e^{-\gamma \Delta t}$. This is equivalent to $e^{\kappa r}$.

As can be seen from Eq. (19), all the Hankel functions in the outgoing wave depend on $r$ through the same factor $\sqrt{\frac{2}{\pi x}} e^{i(k-i \kappa) r}$ in the far-field $(r \gg R)$. Pulling out this common dependence, the field of the quasibound state factorizes into radial and angular functions,

$$
E(\mathbf{r})=\sqrt{\frac{2}{\pi x}} e^{i(k-i \kappa) r} E(\phi) .
$$

This means that the directionality at large distances becomes independent of $r$, being contained solely in $E(\phi)$. We choose $r$ in this far-field region and plot the square of the electric field (which is proportional to the intensity) as a function of $\phi$ to obtain the wave directionality. Figure 16 shows the intensity profile for a resonance of the quadrupole and compares it to the classical directionality histogram for the corresponding ray ensemble. One sees quite reasonable agreement between the full widths of the intensity maxima in the two approaches. The wave intensity distribution is modulated by interference effects which are neglected in the pure ray-optics model (but which should be present in a semiclassical treatment). Moreover the waveintensity envelope does not show the peak splitting found in the ray-optics calculation. It will be interesting to see if resonances at larger $k R$ corresponding to the same $\sin \chi$ begin to show this peak splitting in their envelope. 
Figures available from author's website.

Figure 16: Far-field directionality of light emitted from the quadrupole-deformed cylinder, assuming a refractive index $n=2$ and $\sin \chi_{0}=0.83$. The far-field intensity is plotted as a function of direction at $e=0.58$. The upper curve represents the ray-optics result; the lower curve (offset for clarity) is obtained from exact numerical solution of the wave equation (same resonance as in previous figure).

\section{Thresholds and Intensity Distribution in Lasing Droplets}

In this section we apply the ray-optics model for ARCs to the description of the WG modes of deformed liquid droplets and give an explanation for the observed anisotropy of lasing emission from droplets, shown in Fig. 17 [19]. In this experiment a stream of ethanol droplets containing Rhodamine-B dye with average radius $\approx 30 \mu \mathrm{m}$ is created at the vibrating orifice of a Berglund-Liu generator [35]. At the orifice the droplets are highly non-spherical and as they fall they undergo damped oscillations between oblate and prolate configurations (Fig. 17) driven by excess surface tension until they relax to highly spherical shape far downstream. The period of the shape oscillations is of order $50 \mu \mathrm{s}$, which is far longer than the lifetime of the WG resonances of $\approx 10 \mathrm{~ns}$ [36]. Thus we may treat the different phases of the droplet oscillation as static examples of oblate, spherical or prolate microcavity lasers and analyze the angular emission intensity in terms of the theory of ARCs. Previously we have focused on cylindrical ARCs deformed perpendicular to their axes; here we apply the ray-optics model to dielectric spheres deformed so as to preserve azimuthal symmetry (the deformed droplets retain this symmetry as well as reflection symmetry through the equator to a good approximation).

As seen in Fig. 17, laser emission is fairly isotropic in the sphere, but gets suppressed near the poles of both the oblate and prolate droplets. Furthermore, the oblate shape is brightest around the equator whereas the prolate shape of the same deformation (ratio of long to short axes) emits most strongly from regions around $\theta \approx 30^{\circ}-45^{\circ}$. These observations are stable over a range of prolate and oblate shapes (exceeding a certain degree of deformation) and are independent of the direction of the optical pumping. It is important to note that the three total-energy images are normalized individually with white referring to the maximum emission, which is of different magnitude in each shape.

Since axial symmetry is preserved for the droplets their instantaneous shape can be specified in spherical coordinates by $r_{b}(\theta)$, independent of azimuthal angle $\phi$. The damping is weakest for the low multipole components of the oscillation, so we include only the Legendre polynomials $P_{0}(\cos \theta), P_{2}(\cos \theta), P_{4}(\cos \theta)$ in an expansion of the shape. Odd orders do not appear because of the approximate symmetry $r_{b}(\theta)=$ 
Figures available from author's website.

Figure 17: Shadow graphs (a) and simultaneous total-energy images (b) of three lasing droplets falling in air taken at different phases of oscillation: prolate (top), spherical (middle) and oblate (bottom). Light regions in (b) indicate lasing.

$r_{b}(-\theta)$ noted above. The particular shapes we use to model this behavior more realistically are

$$
r(\theta)=1+\epsilon\left(\cos ^{2} \theta+\frac{3}{2} \cos ^{4} \theta\right)
$$

for prolate deformations; an oblate shape with the same axis ratio [equal to $(5 / 2) \epsilon$ ] is obtained by replacing $\cos \theta$ with $\sin \theta$.

The ray dynamics analysis is facilitated by the axial symmetry of the droplets which implies (in the language of particle trajectories) that the $z$ component of angular momentum, $L_{z}$, is conserved. At any given $L_{z}$ and total energy $E$, the equations of motion thus have only two degrees of freedom, just as in the deformed cylinder. This becomes explicit in cylindrical coordinates $\rho, \phi, z$ where one has

$$
E=\frac{1}{2} m\left(\dot{\rho}^{2}+\dot{z}^{2}\right)+\frac{L_{z}^{2}}{2 m \rho^{2}} .
$$

Let us look at the dynamics projected into the 2D $(\rho, z)$ coordinate system. Each specular reflection causes a discontinuous change in $\dot{\rho}$ and $\dot{z}$; however the angular velocity $\dot{\phi}$ remains unchanged because the normal to the surface of an axisymmetric cavity is always perpendicular to the $\phi$ direction. Thus a 3D specular reflection simply reverses the normal component of the $2 \mathrm{D}$ projected velocity $(\dot{\rho}, \dot{z})$ and reflections are also specular in the projected coordinates. Reflections occur whenever the trajectory $\rho(z)$ intersects the boundary curve $\rho_{b}(z)$. Between reflections the particle motion is free, $\dot{z}=$ const, and Eq. (22) can be integrated to find $\rho(t)$. It can be shown that $\rho^{2}(z)$ describes a parabola whose vertex is the point of closest approach to the $z$-axis and whose intersections with the squared boundary curve $\rho_{b}^{2}(z)$ are the collision points. The curved trajectories in the $z$ - $\rho$-plane between specular bounces [see the inset to Fig. 18(a)] are to be contrasted with the straight paths in conventional $2 \mathrm{D}$ billiards where the centrifugal potential $L_{z}^{2} /\left(2 m \rho^{2}\right)$ is absent. For this reason we call this new class of systems centrifugal billiards.

To discuss the resulting dynamics we introduce dimensionless variables in Eq. (22) by setting $E=1 / 2$ and $m=1$. Then one has

$$
1=\dot{\rho}^{2}+\dot{z}^{2}+\frac{L_{z}^{2}}{\rho^{2}}
$$

where $0 \leq L_{z} \leq \rho_{b}\left(z_{\max }\right)$ if the maximum distance from the $z$-axis is $\rho_{b}\left(z_{\max }\right)$. To simplify notation we assume that the droplets have their widest transverse crosssection in the equatorial plane, i.e. $z_{\max }=0$. Again the escape condition is simply 
Figures available from author's website.

Figure 18: Poincare surfaces of section for prolate droplets with deformation of $\epsilon=0.2$ for $L_{z}=0.735$ (a), $L_{z}=0.6$ (b), $L_{z}=0.45$ (c) and $L_{z}=0$ (d). The dashdotted line denotes the critical line for escape, $\sin \chi_{c}=1 / n=.735$ corresponding to the experimental value of $n=1.36$ for the refractive index of the droplets in Fig. 17. Inset in (a) shows the droplet shape in the $z-\rho$ plane and special (periodic) trajectories for $L_{z}=0.735$ (solid line) and $L_{z}=0.2$ (dashed line).

$\sin \chi<\sin \chi_{c}$, where $\sin \chi$ is the angle of incidence with respect to the surface normal $\mathbf{n}$ at the reflection point. This is not the same as the normal angle in the $\rho-z$-plane, as can be seen by considering a trajectory reflecting entirely in the equatorial plane at nonzero $\sin \chi$; its apparent angle of incidence in the $\rho-z$-plane will be zero. In our units $\cos \chi=\mathbf{n} \cdot \mathbf{v} /|v|=n_{\rho} \dot{\rho}+n_{z} \dot{z}$ since the total velocity is $v=\sqrt{2 E / m}=1$. The angle in the $\rho-z$-plane is then given by $\cos \chi_{\rho z}=\cos \chi / \sqrt{\dot{\rho}^{2}+\dot{z}^{2}}$. It is convenient in the plotting of Poincaré sections to use as variables the polar angle $\theta$ and the 3D $\sin \chi$ at each reflection since in these coordinates the escape condition is still satisfied along a horizontal straight line.

At nonzero $L_{z}$ certain regions of the SOS are forbidden due to the $L_{z}$ angular momentum barrier (e.g. a ray reaching the pole $(\theta=0)$ must have $\left.L_{z}=0\right)$. For the allowed bounce coordinates $\theta, \sin \chi$ one finds the inequality $\sin \chi \geq L_{z} / \rho_{b}(z(\theta))$, where $z(\theta)=r_{b}(\theta) \sin \theta$; this relation delimits the empty spaces in the SOS's of Fig. 18(ad) which were made for a prolate shape of fixed deformation $(\epsilon=0.2)$ and varying values of $L_{z}$. Before discussing ray escape in the deformed droplets it is important to note that as we proceed from higher to lower $L_{z}$ in Fig. 18(a-d) in addition to the excluded regions of the SOS decreasing (because the angular momentum barrier becomes weaker) the degree of chaos grows rapidly. There is actually no visible chaos in Fig. 18(a) and a mostly chaotic SOS for $L_{z}=0$ (Fig. 18(d)) for a droplet of fixed deformation. The reason for this is that high $L_{z}$ trajectories are confined near the equator and a cross-section of the droplet at the equator is perfectly circular, i.e. high $L_{z}$ orbits see an effective deformation which is much weaker than polar orbits $\left(L_{z}=0\right)$ which travel in the most deformed cross-section of the droplet. The effective deformation varies approximately as $\epsilon_{e f f}=\epsilon \sqrt{1-L_{z}^{2} / \rho_{b}^{2}(0)}$ and tends to zero at the maximum allowed value of $L_{z}$. Thus as long as $\epsilon$ is large enough to induce classical Q-spoiling for the $L_{z}=0$ orbits of interest, by looking at different $L_{z}$ values for a fixed deformation one can study the classical Q-spoiling transition in a single ARC. We have illustrated this situation in Fig. 18(a-d).

Note that there is an absolute minimum allowed $\sin \chi \equiv \sin \chi_{m}$ which occurs at the equator $(\theta=\pi / 2)$ where $\rho_{b}$ is maximal (i.e., $\left.\sin \chi_{m}=L_{z} / \rho_{b}(0)\right)$. This implies that classical ray escape is entirely forbidden due to the angular momentum barrier for values of $L_{z} \geq \rho_{b}(0) \sin \chi_{c}$; such a case is shown in Fig. 18(a). As just noted these 
high $L_{z}$ modes are confined to orbits near the plane of the equator [see also the inset to Fig. 18(a)]; since classical escape is forbidden for these modes we always expect to find high-Q WG modes in the equatorial region of axially-symmetric deformed microspheres. Since this follows simply from $L_{z}$ conservation it will be true in both the oblate and prolate shapes.

Proceeding now to lower $L_{z}$ in Fig. 18(b) we see that the angular momentum barrier has weakened enough that the allowed region of the SOS passes through $\sin \chi_{c}$ and rays with this value of $L_{z}$ can escape. However as before WG modes will be associated with rays starting at large $\sin \chi \approx 0.9$ in this case. These rays are unable to reach $\sin \chi_{c}$ due to remaining KAM curves just as we saw earlier in our discussion of dielectric cylinders. Therefore we expect high Q WG modes for this value of $L_{z}$ as well. This situation persists all the way to $L_{z}=0$ for deformations less than roughly $5 \%$ of the radius, so we expect little Q-spoiling and approximately isotropic emission for smaller deformations than this.

However for the $50 \%$ deformation used in Fig. 18(a-d) reducing $L_{z}$ a little more causes the appearance of regions of chaos which extend from high $\sin \chi$ across $\sin \chi_{c}$ allowing classical Q-spoiling of the WG modes. We expect all modes with $L_{z}$ less than this value to have their $\mathrm{Q}$ rapidly degraded. As the $\mathrm{Q}$ of these modes decreases it will fall below the threshold Q-value to support lasing and these modes will go dark. But these low $L_{z}$ modes are the only ones which can emit from the polar regions because of the angular momentum barrier for the high $L_{z}$ modes. Therefore our model explains naturally why the polar regions are dark while the droplet still lases. The low $L_{z}$ modes which could emit from the poles have too low $\mathrm{Q}$ to lase and the high Q modes which support lasing are confined away from the polar regions. This argument holds for both the oblate and prolate deformations in agreement with observations.

We are left with the question of why the emission profiles are nonetheless so different in prolate versus oblate shapes. To answer this question we must look at where the stable islands which block chaotic escape occur for the two types of deformations. The prolate shape corresponds to a stretching of the droplet in the vertical direction and a compression in the equatorial plane. Because it is compressed in the equatorial plane there exists a large stable island at $\theta=\pi / 2$ corresponding to the two-bounce diametral orbit of the type we discussed in the $2 \mathrm{D}$ case in subsection 3.3. This island appears (distorted due to the $L_{z}$ barrier) clearly in Fig. 18(c,d). As discussed above in subsection 6.2, a stable island intersecting the critical line will prevent the classical escape in the corresponding directions; thus in the case of Fig. 18(c) escape is blocked over an interval in polar angle centered at the equator.

In the oblate droplet the situation is reversed. The polar diameter is compressed and the equatorial diameter is stretched and the stable two-bounce orbit (if it is still stable) would appear at $\theta=0, \pi$. But for most $L_{z}$ the island around this orbit is unreachable and it has little effect on the dynamics. In Fig. 19(a) and (b) the SOS for the prolatee and oblate shapes are compared at equal deformation and equal 
Figures available from author's website.

Figure 19: Prolate (a) and oblate (b) droplet SOS's at $\epsilon=0.2$ and $L_{z} / \rho_{b}(0)=0.3$. As in the previous Figure, the dash-dotted lines indicate $\sin \chi_{c}$. The escape directionality is shown in (c) for the prolate (filled histogram) and oblate (white) shape.

ratio $L_{z} / \rho_{b}(0)$. Indeed in the SOS for the oblate shape the regular island centered on $\theta=\pi / 2$ and $\sin \chi_{m}$ is absent because equatorial orbits with low $\sin \chi$ are now unstable. One sees no effect of the stable islands at $\theta=0, \pi$ due to the angular momentum barrier. There is still an island near the critical line at $\theta=0$ and $\sin \chi=0.6$ for this particular $L_{z}$, but its origin (a three-bounce orbit in the $\rho-z$ plane) as well as its effect are quite different from the prolate shape. In fact, all downward-diffusing trajectories first cross $\sin \chi_{c}$ in the vicinity of this island because phase-space flow (see subsection 6.2) roughly follows a V-shaped curve connecting the three islands located at $\sin \chi=0.825(\theta= \pm 0.5)$ and $\sin \chi=0.6(\theta=0)$. Hence escape is concentrated in the equatorial region. This remains true even if the island actually intersects the critical line (as is the case for higher $L_{z}$ ), thus blocking escape right at $\theta=0$ - in that case escape still occurs very close to the island.

Our explanation for the differing intensity profiles is as follows. First, it is reasonable to assume that all modes for which classical escape is completely forbidden will lase. As $L_{z}$ decreases and classical escape begins to occur there will exist modes which are lower Q, but still high enough $\mathrm{Q}$ to lase. These modes will have highly directional emission in the polar angle (they will of course emit uniformly in the azimuthal angle). In the prolate case as $L_{z}$ decreases the first regions of chaos which connect the WG orbits to the critical line do so only in small intervals around $\theta=30^{\circ}$ and $\theta=150^{\circ}$ (see Figs. 18(c) and 19(a)). These values of $L_{z}$ will correspond to the lower Q lasing modes just discussed and will thus emit in small bright bands around these latitudes. In contrast, in the oblate droplets phase space flow curves touch the critical line near the equator (see Fig. 19(b)) and the lower Q lasing modes will emit around $\theta=0$. However the total lasing intensity will be due to all the lasing modes and there will typically also be many high $L_{z}$ modes of higher $\mathrm{Q}$ which can emit at the equator through the same processes as in the undeformed droplet. But unlike the undeformed case, these modes are competing with lower Q lasing modes corresponding to lower $L_{z}$ which therefore have higher gain and capture more of the pump energy. Since more of the pump energy goes into these highly anisotropic modes whose emission pattern differs strongly between the prolate and oblate shapes, these shapes show essentially the intensity profiles predicted by the ray-optics model for the intermediate values of $L_{z}$ corresponding to slow classical escape and therefore low but above-threshold $Q$ values.

To produce a numerical simulation of the cumulative directionality of all the lasing modes with classical escape, we consider ensembles of starting conditions ho- 
mogenously distributed in $\phi$ as in section 6. If the average $Q$ of an ensemble starting at some $\sin \chi_{0}$ is above the lasing threshold, we record the resulting escape directionality; otherwise we discard that particular ray bundle. Repeating this for uniformly spaced $\sin \chi_{0} \in\left[\sin \chi_{c}, 1\right]$ and $L_{z} / \rho(0) \in[0,1]$, one obtains Fig. 19(c). It can be seen that maximum emission occurs near the equator for the oblate shape, and around $30^{\circ}$ away from the poles in the prolate droplet. The emission peak of the oblate droplet is split because of the island shown in Fig. 19(b), but this structure is not seen in Fig. 17. Nevertheless, the ray theory is clearly able to account for the overall location of the emission maxima seen experimentally. The discrepancies in fine structure may be resolvable by modifying the parametrization in Eq. (21) which we chose to model the shape. More experiments are required to check if the above splitting does indeed occur in droplets at different oscillation stages.

\section{Summary and Conclusions}

In this chapter we have begun to develop a theory of a new class of asymmetric resonant cavities (ARCs). A large class of deformations of dielectric spheres or cylinders were shown to lead to resonators with similar properties. This universality can be understood in terms of partially chaotic ray dynamics described by KAM theory which we have incorporated into a ray-optics model for the whispering gallery modes of ARCs. The $\mathrm{Q}$ of these modes decreases sharply at a threshold deformation and above this deformation the emission is very directional. The theory provides a natural explanation for the anisotropic lasing intensity profile of deformed lasing droplets. Systematic experimental investigation of ARCs has just begun, and only for the case of lasing droplets; further experiments are of paramount importance at this point. ARCs may provide tests and practical applications for ideas from KAM theory and quantum chaos. We have proposed a number of potential applications of ARCs which we conjecture may combine the advantages of microsphere and microdisk cavities with the directional properties of Fabry-Perot cavities. The feasibility of these applications cannot be judged unless significant effort is put into the experimental study of solid ARCs.

\section{Acknowledgements}

We would like to acknowledge the major contribution of Attila Mekis to the section on lasing droplets and ray directionality, as well as valuable discussions with Henrik Bruus and Dima Shepelyansky. We have also benefited greatly from close contact and collaboration with Richard Chang and his experimental group, particularly Gang Chen. This work was partially supported by the NSF under grant no. DMR-9215065. 


\section{References}

[1] Y. Yamamoto and R.E. Slusher, Physics Today 46, 66 (1993).

[2] S. L. Mcall et al. , Appl. Phys. Lett. 60, 289 (1992).

[3] R.E. Slusher et al. , Appl. Phys. Lett. 63, 1310 (1993).

[4] M. Kuwata-Gonokami et al. , QELS Technical Digest '95, Paper QThL5, Polymer microdisk and microcylinder lasers; M. Kuwata-Gonokami et al., preprint.

[5] A. Serpengüzel, S. Arnold and G. Griffel, Opt. Lett. 20, 654 (1995), and references therein.

[6] S. C. Hill and R. K. Chang in Proceedings of the 5th International Topsøe Summer School on Nonlinear Optics (Aalborg, Denmark), O. Keller, ed. (Nova Science Publishers, New York, 1992)

[7] L. Collot V. Lefevre-Seguin, M. Raimond and S. Haroche, Europhys. Lett. 23, 327 (1993).

[8] The term whispering gallery mode has been adopted in optics because of the similarity of these modes to analogous modes in acoustics which were first understood by Lord Rayleigh. These modes have high-energy density only in a small annulus at the perimeter of the cavity and scatter very little so that in an acoustic cavity (e.g. St. Paul's cathedral) it is possible to whisper and be heard clearly at the opposite side of the cavity, but not in its center. In the context of the optical study of microdroplets these modes are often referred to as "morphology-dependent resonances" (MDR's). In this chapter we shall use the term whispering gallery mode to refer to all such modes.

[9] E. S. C. Ching, P. T. Leung, K. Young, Chapter 1 of this volume.

[10] The only relevant exception is the case of elliptical deformations (discussed in subsection 3.2) which may be treated analytically, see [C. Yeh, J. Math. Phys. 4, 65 (1963)] but which is not typical because the ray dynamics remains integrable for all deformations.

[11] P. Barber and C. Yeh, Appl. Opt. 14, 2864 (1975)

[12] D. R. Wilton and R. Mittra, IEEE Trans. Ant. Propagation AP-20, 310 (1972)

[13] M. C. Gutzwiller, Chaos in Classical and Quantum Mechanics (Springer, New York 1990)

[14] H. U. Baranger, R. A. Jalabert and A. D. Stone, CHAOS, 3, 665 (1993). 
[15] G. Casati, B. V. Chirikov, I. Guarneri and D. L. Shepelyanski, Phys. Rev. Lett. 56, 2437 (1986)

[16] It is amusing to note an analogy between the microwave ionization of hydrogen and our current topic. The dielectric microspheres which trap a long-lived photon are sometimes referred to as "photonic atoms"; thus one could say we are studying the classical "ionization" of the photonic atom due to a perturbation of its shape.

[17] J. U. Nöckel, A. D. Stone and R. K. Chang, Opt. Lett. 19, 1693 (1994).

[18] A. F. Levi et al. , Appl. Phys. Lett. 62, 561 (1993)

[19] A. Mekis, J. U. Nöckel, G. Chen, A. D. Stone and R. K. Chang, to be published in Phys. Rev. Lett.

[20] B. R. Johnson, J. Opt. Soc. Am. 10, 343 (1993).

[21] A. N. Kolmogorov, Dokl. Akad. Nauk. SSSR 98, 527 (1954); J. Moser, Nachr. Akad. Wiss. Göttingen, Math. Phys. Kl. , 1 (1962); V. I. Arnol'd, Russian Math. Surveys 18, 9 (1963); see the discussions in Ref. 23 and [A. M. Ozorio de Almeida, Hamiltonian Systems: Chaos and Quantization (Cambridge University Press, Cambridge, 1988)].

[22] V. F. Lazutkin, Math. USSR Izvestija 7, 185 (1973)

[23] L.E. Reichl, The Transition to Chaos, (Springer-Verlag, Berlin, 1992)

[24] M. Robnik, J. Phys. A 16, 3971 (1983); M. Robnik, J. Phys. A 17, 1049 (1983).

[25] M. V. Berry, Eur. J. Phys. 2, 91 (1981)

[26] H. Poincaré, Les Méthodes Nouvelles de la Méchanique Céleste Tome III, (Gauthier-Villars, Paris, 1899)

[27] D. Q. Chowdhury, P. W. Barber and S. C. Hill, Appl. Opt. 313518 (1992)

[28] L.P. Kadanoff, Phys. Rev. Lett. 47, (1981); D. Bensimon and L. P. Kadanoff, Physica D 13, 82 (1984); R. S. MacKay, J. D. Meiss, I. C. Percival, Physica D 13, 55 (1984); R. S. MacKay, J. D. Meiss, I. C. Percival, Phys. Rev. Lett. 52, 697 (1984) (1984).

[29] L.P. Kadanoff, J. Stat. Phys. 27, 631 (1981)

[30] See the articles in the focus issue on "Chaotic Scattering", CHAOS 3 (1993).

[31] J. U. Nöckel, A. Mekis and A. D. Stone, unpublished 
[32] O. Bohigas, S. Tomsovic and D. Ullmo, Phys. Rep. 223, 43 (1993)

[33] J. B. Keller and S. I. Rubinov, Ann. Phys. 9, 24 (1960)

[34] J. U. Nöckel and A. D. Stone, unpublished

[35] S. X. Qian, J. B. Snow, H. M. Tzeng, and R. K. Chang, Science 231, 486 (1986).

[36] J. Z. Zhang, D. H. Leach and R. K. Chang, Opt. Lett. 13, 270 (1988) 